\title{
Metabolic substrate utilization in stress-induced immune cells
}

\author{
Xiaomin Zhang ${ }^{1 \dagger}$, Fabian Zink ${ }^{{ }^{*}+}$ (D), Felix Hezel ${ }^{1}$, Josef Vogt ${ }^{1}$, Ulrich Wachter ${ }^{1}$, Martin Wepler ${ }^{1,2}$, Maurizio Loconte ${ }^{3}$,
} Christine Kranz ${ }^{4}$, Andreas Hellmann ${ }^{4}$, Boris Mizaikoff ${ }^{4}$, Peter Radermacher ${ }^{1}$ and Clair Hartmann ${ }^{1,2}$

From 4th International Symposium on Acute Pulmonary Injury and Translational Research - INSPIRES 2019

Dresden, Germany. 25-26 November 2019

\author{
* Correspondence: fabian.zink@uni- \\ ulm.de \\ ${ }^{+}$Xiaomin Zhang and Fabian Zink \\ contributed equally to this work. \\ ${ }^{1}$ Institut für Anästhesiologische \\ Pathophysiologie und \\ Verfahrensentwicklung, \\ Universitätsklinikum UIm \\ Helmholzstraße 8/1, 89081 Ulm, \\ Germany \\ Full list of author information is \\ available at the end of the article
}

\begin{abstract}
Immune cell activation leads to the acquisition of new functions, such as proliferation, chemotaxis, and cytokine production. These functional changes require continuous metabolic adaption in order to sustain ATP homeostasis for sufficient host defense. The bioenergetic demands are usually met by the interconnected metabolic pathways glycolysis, TCA cycle, and oxidative phosphorylation. Apart from glucose, other sources, such as fatty acids and glutamine, are able to fuel the TCA cycle.

Rising evidence has shown that cellular metabolism has a direct effect on the regulation of immune cell functions. Thus, quiescent immune cells maintain a basal metabolic state, which shifts to an accelerated metabolic level upon immune cell activation in order to promote key effector functions.

This review article summarizes distinct metabolic signatures of key immune cell subsets from quiescence to activation and demonstrates a methodical concept of how to assess cellular metabolic pathways. It further discusses why metabolic functions are of rising interest for translational research and how they can be affected by the underlying pathophysiological condition and/or therapeutic interventions.
\end{abstract}

Keywords: Immunometabolism, Glycolysis, Pentose phosphate pathway, Tricarboxylic acid cycle, Oxidative phosphorylation, Reactive oxygen species, Catecholamines

\section{Introduction}

Immune cell responses in inflammation are highly dynamic and require continuous metabolic adaption in order to maintain a sufficient host defense. The bioenergetic demands are usually met by the interconnected metabolic pathways glycolysis, tricarboxylic acid (TCA) cycle, and oxidative phosphorylation (OXPHOS). Briefly, during glycolysis, glucose is converted into pyruvate in the cytoplasm and enters the mitochondria after the conversion to acetyl-coenzyme A (-CoA). In the TCA cycle, acetyl-CoA is degraded, resulting in nicotinamide adenine dinucleotide $(\mathrm{NADH})$ and flavin adenine dinucleotide $\left(\mathrm{FADH}_{2}\right)$ formation, which serve as electron donors for OXPHOS, ultimately resulting in adenosine triphosphate (ATP) generation. As a natural byproduct of the electron transport within the respiratory chain, reactive oxygen species (ROS) are produced alongside OXPHOS. Apart

(c) The Author(s). 2021, corrected publication 2021. Open Access This article is licensed under a Creative Commons Attribution 4.0 International License, which permits use, sharing, adaptation, distribution and reproduction in any medium or format, as long as you give appropriate credit to the original author(s) and the source, provide a link to the Creative Commons licence, and indicate if changes were made. The images or other third party material in this article are included in the article's Creative Commons licence, unless indicated otherwise in a credit line to the material. If material is not included in the article's Creative Commons licence and your intended use is not permitted by statutory regulation or exceeds the permitted use, you will need to obtain permission directly from the copyright holder. To view a copy of this licence, visit http://creativecommons.org/licenses/by/4.0/. 
from glucose, other sources, such as fatty acids and glutamine, are able to fuel the TCA cycle. Fatty acids enter the TCA cycle after being subjected to $\beta$-oxidation to generate acetyl-CoA. Glutamine is converted to glutamate via glutaminolysis and enters the TCA cycle after being transformed to $\alpha$-ketoglutarate.

It is well-known that under hypoxic conditions ATP is generated via anaerobic glycolysis by converting pyruvate to lactate. Intriguingly, some types of immune cells show a similar metabolism even when oxygen availability is not limited. These cells preferentially use aerobic glycolysis for ATP production, a metabolic pathway which was initially reported in cancer cells by Warburg et al. [1]. It is of course undisputable that, regarding the ATP yield, aerobic glycolysis ( $2 \mathrm{~mol} \mathrm{ATP} / \mathrm{mol}$ glucose) is far less efficient compared to OXPHOS ( $\approx 36 \mathrm{~mol} \mathrm{ATP} / \mathrm{mol}$ glucose); however, this difference can be compensated for by a high speed of aerobic glycolysis [2]. A high flux through aerobic glycolysis subsequently increases the flux through the pentose phosphate pathway (PPP), a metabolic shunt that parallels glycolysis. The PPP generates five-carbon sugars for nucleotide synthesis and nicotinamide adenine dinucleotide phosphate (NADPH), crucial for NADPH-dependent oxidative burst and fatty acid biosynthesis. Figure 1 summarizes core metabolic pathways of immune cell subsets, which will be discussed in the following manuscript.

As mentioned above, biological functions of immune cells are tightly linked to metabolic programs, which are highly plastic and adapt to promote their changing functions. This review article summarizes distinct metabolic signatures of key immune cells from quiescence to activation and discusses their potential role as bioenergetic biomarkers in translational research.

\section{Distinct energetic profiles of immune cells}

In general, immune responses are composed of the innate and the adaptive immune system. Upon encountering with a pathogen, the innate immune response, which is composed of neutrophils, monocytes/macrophages, and natural killer (NK) cells, represents the first stage and is nonspecific and rapid. The second stage is defined by the adaptive branch of the immune response, which is composed of a small fraction of cells highly specific for any pathogen/antigen and comprises T cells and B cells. An immunological memory is not only known for the adaptive immune system but has previously also been attributed to the cells of the innate immune system, also referred to as trained immunity. Trained immunity is particularly triggered by pattern recognition receptors, which are expressed on innate immune cells in order to recognize pathogen molecules and is regulated by metabolic and epigenetic reprogramming [3,4]. Thus, similarly to the known immune responses, trained immunity induces changes in intracellular immune signaling by rewiring the energy metabolism. This leads to an enhanced proinflammatory response upon a subsequent inflammatory challenge eventually improving survival of the host. In contrast to the adaptive immune system, innate immune responses are unspecific due to a lacking antibody-antigen interaction.

Regarding the energy metabolism, quiescent immune cells maintain a basal metabolic state, which shifts to a higher metabolic level upon immune cell activation in order to promote key effector functions. The cell type-specific differences of ATP-producing pathways in quiescence and upon activation will be outlined below (Fig. 2). 


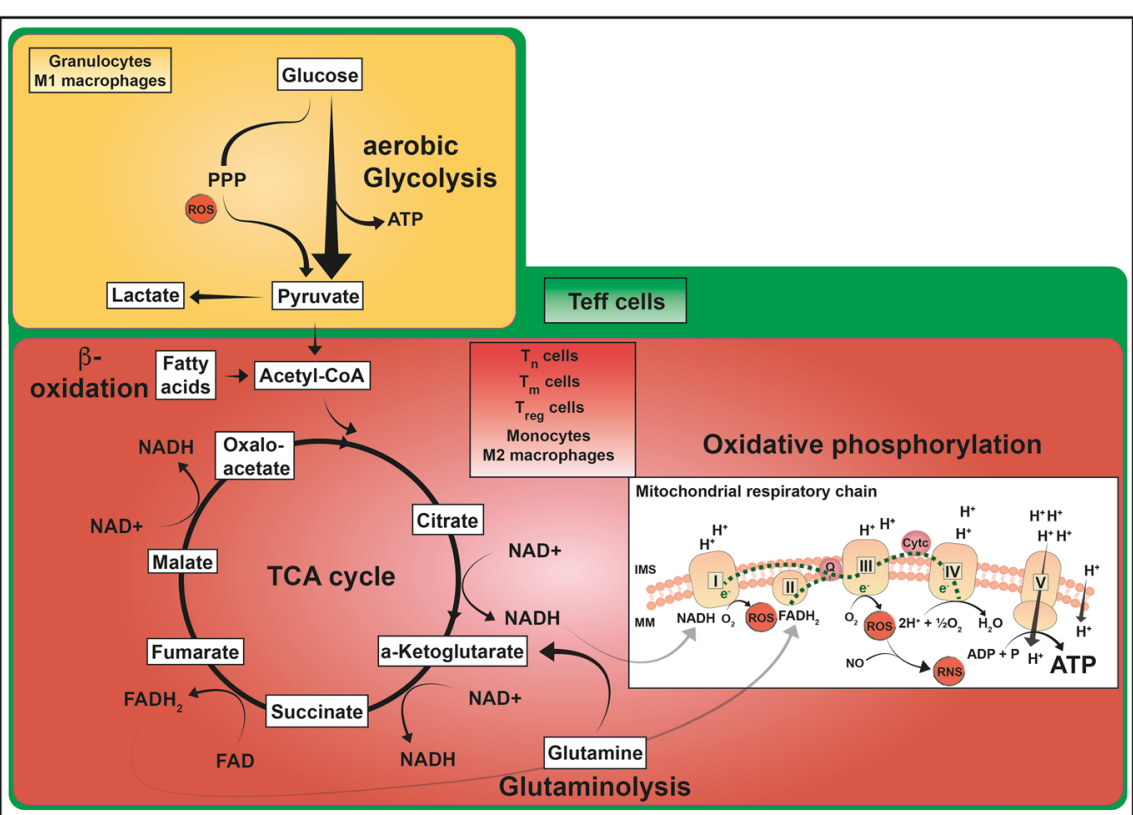

Fig. 1 ATP-producing metabolic pathways in distinct immune cell subsets. Glucose oxidation to pyruvate via glycolysis is a fast reaction generating $2 \mathrm{~mol}$ of ATP per mol glucose. This aerobic glycolysis is complemented by the PPP that can produce further metabolic precursor molecules and is involved in ROS production. Pyruvate can be converted to lactate or can be further oxidized to acetyl-CoA entering the mitochondrial TCA cycle (yellow box). The TCA cycle (red box) generates reducing equivalents $\mathrm{NADH}$ and $\mathrm{FADH}_{2}$ which are utilized in the mitochondrial respiratory chain to build up the proton gradient across the mitochondrial inner membrane by complexes I-IV of the respiratory chain. As a by-product, ROS and RNS are produced. Oxidative phosphorylation produces larger amounts of ATP (36-38 mol/mol glucose) by complex V. Immune cells are also able to utilize substrates such as glutamine, which enters these pathways via the TCA metabolite a-ketoglutarate, and fatty acids, which are oxidized to acetyl-CoA via $\beta$-oxidation. Granulocytes and M1 macrophages have a highly glycolytic metabolism (yellow box) even when oxygen is available. Their TCA cycle and respiratory chain activity is kept at low level. $T_{n}, T_{m}$, and $T_{\text {reg }}$ cells as well as monocytes and M2 macrophages primarily perform OXPHOS and are also able to metabolize fatty acids and glutamine in order to fuel the TCA cycle (red box). Teff cells have a highly active metabolism including all of the pathways described (green box). These pathways do not only culminate in ATP production but also provide other biosynthetic pathways with metabolic precursors thus supporting different functional necessities of the immune cell populations. Abbreviations: ADP, adenosine diphosphate; ATP, adenosine triphosphate; $C \mathrm{CA}$, coenzyme $\mathrm{A} ; \mathrm{FADH} 2 / \mathrm{FAD}$, flavin adenine dinucleotide in its reduced/oxidized form; IMS, intermembrane space; $\mathrm{MM}$, mitochondrial membrane; $\mathrm{NADH} / \mathrm{NAD}^{+}$, nicotinamide adenine dinucleotide in its reduced/oxidized form; OXPHOS, oxidative phosphorylation; PPP, pentose phosphate pathway; ROS, reactive oxygen species; RNS, reactive nitrogen species; TCA, tricarboxylic acid cycle; $T_{\text {eff, }}$ effector T cell; $T_{n}$, naive $T$ cell; $T_{m}$, memory $T$ cell; $T_{\text {reg, }}$, regulatory $T$ cell

\section{Granulocytes}

Neutrophils only have a few mitochondria, primarily important for mitochondrial membrane potential maintenance and apoptosis initiation $[5,6]$. Neutrophils consume low amounts of oxygen [7] and produce ATP through aerobic glycolysis [8-10]. Metabolic profiles of granulocytes have mostly been described in neutrophils; however, there is some evidence showing that basophilic and eosinophilic granulocytes depend on glycolytic metabolism [11]. Besides, Porter et al. demonstrated that human eosinophils exhibited a higher basal oxygen consumption rate and reserve respiratory capacity, thereby allowing them to be metabolically more versatile [12].

After the recruitment to an inflammatory tissue site in response to pro-inflammatory stimuli, neutrophils become fully activated, a condition of diverse functions including oxidative burst, the production of neutrophil extracellular traps (NETs), and 


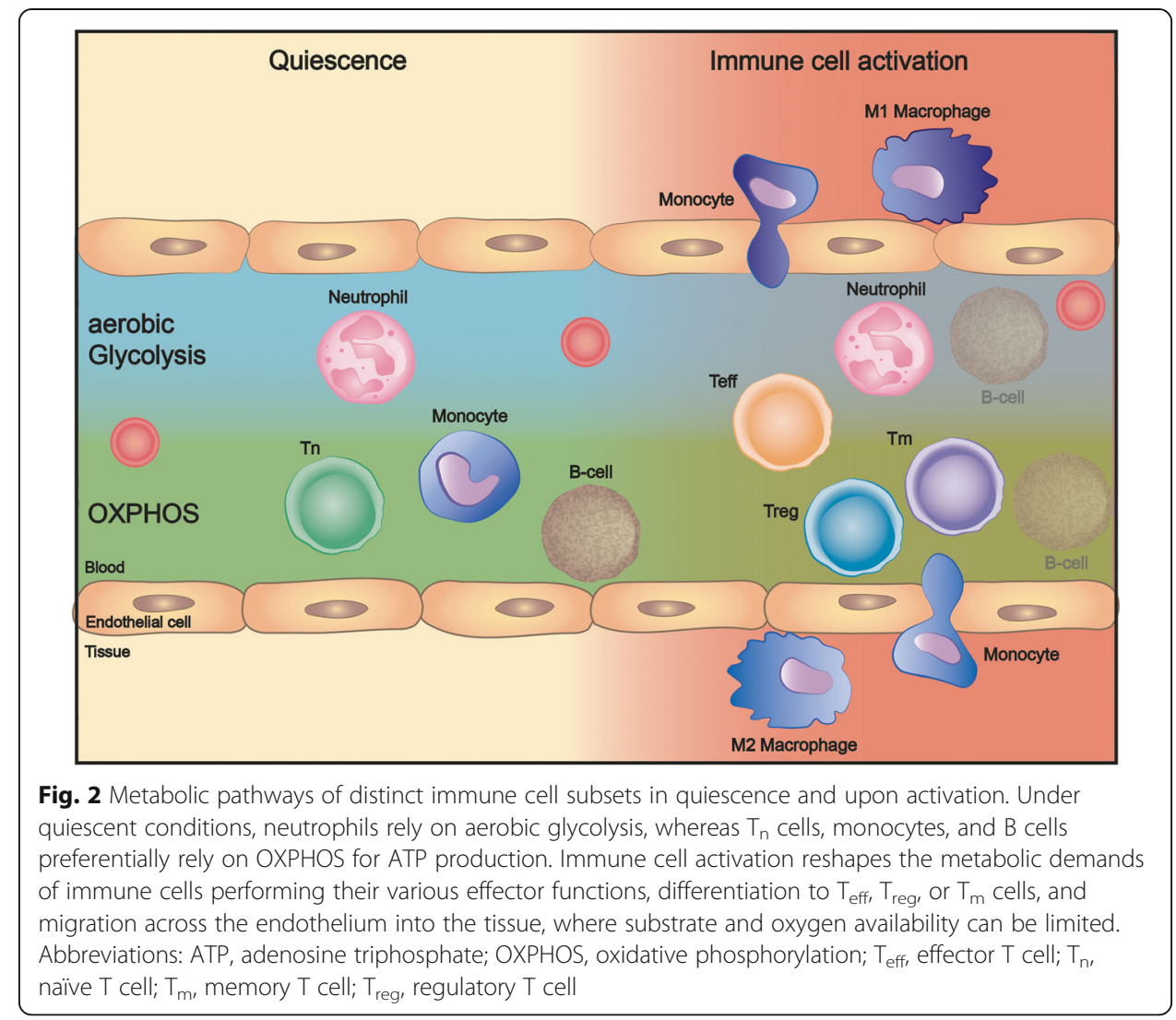

phagocytosis. In this state, neutrophils require large amounts of ATP, which is primarily produced via aerobic glycolysis $[13,14]$. This oxygen-independent metabolism is particularly beneficial when neutrophils are recruited to inflammatory sites with low oxygen partial pressure.

Hypoxia and inflammation are tightly related: hypoxia may be the consequence of inflammatory processes or may vice versa actively promote inflammatory responses. In both cases, it is necessary that immune cells sense and adapt to low oxygen levels in order to continue to perform their cell type-specific functions under these conditions. The metabolic adaption to hypoxia is primarily mediated by hypoxia-inducible transcription factors (HIFs), a family of transcription factors with oxygen-sensitive $\alpha$-subunits. However, HIFs can also be activated by an inflammatory reaction thereby upregulating glycolytic gene expression to safeguard elevated ATP requirements via aerobic glycolysis and by generating valuable precursors for cellular proliferation and effector function, e.g., nucleotides, lipids, and amino acids [15-17]. The effects of HIFs strongly depend on the cell type. In myeloid cells, which are primarily responsible for innate immune defenses, HIF1 $\alpha$ promotes pro-inflammatory effects by increasing pathogen clearance features, such as motility, invasiveness, and bactericidal activity [18]. In the adaptive immune system, HIF1 $\alpha$ promotes the differentiation of regulatory $\mathrm{T}$ cells $\left(\mathrm{T}_{\text {reg }}\right)$, thereby increasing anti-inflammatory and tissue-protective effects [19]. Thus, HIF1 $\alpha$ specifically modulates the metabolism of distinct immune cell subsets in order to generate a balanced and controlled immune response [20]. 
In neutrophils, adequate intake of glucose is particularly mediated by the glucose transporter-1 (GLUT-1) and GLUT-3 [21], while HIF1 $\alpha$ maintains glycolysis by upregulating genes of key glycolytic enzymes and at the same time repressing genes for OXPHOS [18]. Glycolysis is crucial for neutrophilic functions, such as phagocytosis and NET formation, a mechanism to kill microorganisms by releasing chromatin traps, as shown in phorbol myristate acetate (PMA)-stimulated human neutrophils [22]. Furthermore, other authors highlighted the importance of the PPP in NET formation, arising mainly from NADPH oxidase (NOX)-derived ROS and further highlighted the importance of mammalian target of rapamycin (mTOR) regulating HIF1 $\alpha$ in this context [23, 24].

Upon neutrophil activation, a high flux through the glycolytic pathway subsequently increases the flux through the PPP, in order to generate NADPH, which is in turn used by NOX to generate superoxide anions $\left(\mathrm{O}_{2}^{-}\right)$[25-27]. This mechanism is also referred to as the "oxidative burst." Guthrie et al. demonstrated that human lipopolysaccharide (LPS)stimulated neutrophils increased their glucose uptake and oxygen consumption, which, however, was used for hydrogen peroxide $\left(\mathrm{H}_{2} \mathrm{O}_{2}\right)$ production rather than OXPHOS [28].

Last, due to their metabolic plasticity, neutrophils are able to meet their energy requirements during differentiation processes and/or under glucose-limiting conditions by utilizing other metabolic substrates, such as fatty acids via $\beta$-oxidation and glutamine via glutaminolysis [29-31].

\section{Monocytes}

Monocytes are crucial effectors and regulators of innate immune response. Resting monocytes do not proliferate and remain in a low metabolic steady state driven by oxidative metabolism [32].

Upon an inflammatory stimulus, activated monocytes eliminate pathogens via phagocytosis, ROS, and cytokine production, among others, by shifting their energy metabolism to a glycolytic phenotype, mediated by mTOR-HIF1 $\alpha$, in order to satisfy higher ATP requirements $[33,34]$. Besides, monocytes are recruited to the inflammatory tissue and continue to differentiate into two different subtypes: M1 macrophages, promoting a pro-inflammatory response [35], or M2 macrophages, which are anti-inflammatory and play a vital role in modulating inflammation and repairing tissue [36].

These highly distinct functional phenotypes are reflected by opposing metabolic requirements. The pro-inflammatory M1 condition depends on aerobic glycolysis and thus shows low oxygen consumption rates [34, 37, 38]. Glucose uptake is enhanced by the increased expression of GLUT1-3 as demonstrated in PMA- or LPS-stimulated human monocytes, but also in T and B lymphocytes [21]. Similarly, as shown in neutrophils, HIF $1 \alpha$ is crucially involved in promoting/maintaining a glycolytic metabolism [39]. However, macrophages are not only able to utilize glucose but also glutamine as demonstrated in activated murine peritoneal macrophages by Newsholme et al. [40].

During the resolution of inflammation, macrophages differentiate into the M2 phenotype, which primarily performs OXPHOS metabolism fueled by fatty acid oxidation (FAO) [41]. In fact, after inhibition of OXPHOS, M2 macrophage expression was attenuated, and, moreover, they were forced into the M1 macrophage phenotype [32, 42]. In other words, the functional (M1 vs. M2) state of monocytes coincides with a timedependent sequence of metabolic activity, with enhanced glycolysis and PPP turnover 
during the activation phase and back to TCA and OXPHOS, respectively, during the deactivation phase i.e. the resolution phase of inflammation [43, 44].

Monocytes have extensively been studied in respect to trained immunity. Arts et al. elegantly demonstrated that $\beta$-glucan-induced trained immunity in human monocytes was mediated by profound rewiring of cellular metabolism [45]. Thus, particularly glycolysis, glutaminolysis and cholesterol synthesis were described as key metabolic pathways. Besides, the authors identified the TCA metabolite fumarate as a crucial player in promoting epigenetic reprogramming [45]. Similarly, other studies in human $\beta$-glucan trained monocytes reported an increase of particularly glycolytic genes primarily mediated by the Akt/mTOR/HIF1 $\alpha$ pathway [46].

\section{Lymphocytes}

\section{$T$ lymphocytes}

Unstimulated naïve T lymphocytes primarily use OXPHOS to generate ATP [10, 47]. Subsequently to antigen recognition and co-stimulation, activated $\mathrm{T}$ lymphocytes rapidly grow and differentiate into subpopulations, such as effector $T$ cells $\left(\mathrm{T}_{\text {eff }}\right), \mathrm{T}_{\text {reg, }}$, and memory $\mathrm{T}$ cells $\left(\mathrm{T}_{\mathrm{m}}\right)$. This developmental program requires large amounts of energy in order to generate a sufficient amount of ATP [48-50]. $\mathrm{T}_{\text {eff }}$ cells are crucial players during an inflammatory response. They have both immune promoting but also negative regulatory effects thereby steering immune responses. $\mathrm{T}_{\text {eff }}$ cells have a reduced mitochondrial mass and a low reserve respiratory capacity and generate ATP predominantly through aerobic glycolysis over OXPHOS [51]. This coincides with increased GLUT-1 expression and glucose uptake [52-55]. Apart from a high glycolytic rate, $\mathrm{T}_{\text {eff }}$ show increased biosynthetic activity by promoting nucleotide synthesis via PPP.

In contrast, $\mathrm{T}_{\text {reg }}$ cells and $\mathrm{T}_{\mathrm{m}}$ cells are non-proliferative cells and adopt OXPHOS and FAO for ATP generation [56-58]. $\mathrm{T}_{\text {reg }}$ cells are a specialized subpopulation of lymphocytes that suppress immune responses to balance pro-inflammation. According to Angelin et al., $\mathrm{T}_{\text {reg }}$ cells adopt oxidative metabolism induced by forkhead box protein 3 (FoxP3), which can downregulate glycolysis in mouse models including colitis, cardiac allografting, and homeostatic proliferation [59]. Consistent with this notion, Gerriets et al. found $\mathrm{T}_{\text {reg }}$ cells to also display aerobic glycolysis when FoxP3 is reduced in a murine model [60]. $\mathrm{T}_{\mathrm{m}}$ embody features of both naive and effector cells. Bioenergetically, $\mathrm{T}_{\mathrm{m}}$ cells have more mitochondrial mass and a higher reserve respiratory capacity compared to naïve cells [61]. They are metabolically primed and thus are able to rapidly respond when the same pathogen attacks the host [57].

\section{B lymphocytes}

B cells are a critical part of the humoral immunity e.g. by secreting antibodies and promote $\mathrm{T}$ cell activation [62]. However, data on $\mathrm{B}$ cell lymphocyte metabolism is relatively scarce, as the previously mentioned studies primarily concentrated on $\mathrm{T}$ lymphocytes. Recent evidence showed that resting B cells seem to have lower energy requirements than resting $\mathrm{T}$ cells as they consumed less glucose and fatty acids and, consequently, produced less ATP [63]. Nevertheless, resting B cells primarily rely on OXPHOS to meet their metabolic demands and have a higher mitochondrial mass [63]. Despite these differences in the resting state, B cells share some metabolic 
characteristics with $\mathrm{T}$ cells upon activation. Thus, these cells rapidly increase glucose uptake following cell antigen receptor activation; phosphatidylinositol 3-kinase (PI-3 K) is indispensable to increase glucose utilization $[64,65]$. In line with this, Limon et al. showed that B cells largely depend on glycolysis for proliferation [66]. However, recent research from Waters et al. displayed different notions that activated B cells upregulated OXPHOS rather than glycolysis, despite increased glucose uptake during B cell activation [67].

\section{A concept to assess immune cell metabolism}

The variable substrate utilization of immune cells as well as their impact on energy metabolism can be assessed ex vivo by the following methodical approach: (1) Metabolic flux analysis is performed by incubating immune cells with stable, non-radioactive isotope-labeled substrates (e.g., parallel incubation with ${ }^{13} \mathrm{C}$-glucose, ${ }^{13} \mathrm{C}$-glutamine [68]). Subsequently, measurements of the isotope enrichment in various metabolites and cleavage products of the glycolytic pathway, the PPP, and the TCA-cycle are performed, followed by conversion of labeling patterns to estimate relative pathway activities and by quantification of ${ }^{13} \mathrm{CO}_{2}$ release from the respective isotopes (Fig. 3) [69-71]. (2) Measurement of mitochondrial oxygen consumption and (3) production of ROS (Fig. 4).

We recently showed in a long-term, resuscitated porcine model of acute subdural hematoma (ASDH) combined with hemorrhagic shock that the mitochondrial oxygen consumption was more pronounced in peripheral blood mononuclear cells (PBMCs) than in granulocytes in both the quiescent (MP1, measuring point) and the activation state $(\mathrm{MP} 3+4)($ Fig. 4a, unpublished data). In contrast, granulocytes had a several-fold higher production of both the $\mathrm{O}_{2}^{-}$(Fig. 4b, adapted from [72]) and the reactive species $\mathrm{H}_{2} \mathrm{O}_{2}$ (Fig. 4c, adapted from [72]). In respect to the isotope data, we could recently demonstrate in a long-term, resuscitated porcine ASDH-induced acute brain injury model [73] that PBMC-related ${ }^{13} \mathrm{CO}_{2}$ production from glutamine was approximately five times higher than that of glucose-derived ${ }^{13} \mathrm{CO}_{2}$ (Fig. 3b, unpublished data). In line with our findings, Fig. $4 \mathrm{~d}$ reflects the concept for this methodical approach of analyzing the energy metabolism in distinct immune cell populations. According to their ability of metabolizing glutamine (glutaminolysis), ${ }^{13} \mathrm{CO}_{2}$ production from glutamine is higher in PBMCs compared to ${ }^{13} \mathrm{CO}_{2}$ derived from glucose, due to their low rate of aerobic glycolysis (Fig. 4d). PBMCs primarily perform OXPHOS in order to produce ATP, which is reflected by a high rate of mitochondrial oxygen consumption. In contrast, granulocytes generate ATP via aerobic glycolysis, resulting in low oxygen consumption rates (Fig. 4d). A high flux through the glycolysis pathway is accompanied by a high flux through the PPP leading to higher production rates of ROS in granulocytes compared to PBMCs (Fig. 4d).

\section{The role of reactive oxygen species and catecholamines}

As mentioned above, ROS are a natural byproduct of the electron transport within the respiratory chain. Although early literature from Boveris et al. estimated that about $1-2 \%$ of the total consumed oxygen was diverted to $\mathrm{H}_{2} \mathrm{O}_{2}$ production in a steady state [74], recent findings take a more comprehensive approach into account. Thus, the rate of mitochondrial $\mathrm{O}_{2}{ }^{-}$and $\mathrm{H}_{2} \mathrm{O}_{2}$ production depends on the cell type, on substrate availability, the source of reducing equivalents, and on (patho-)physiological states $[75,76]$. 


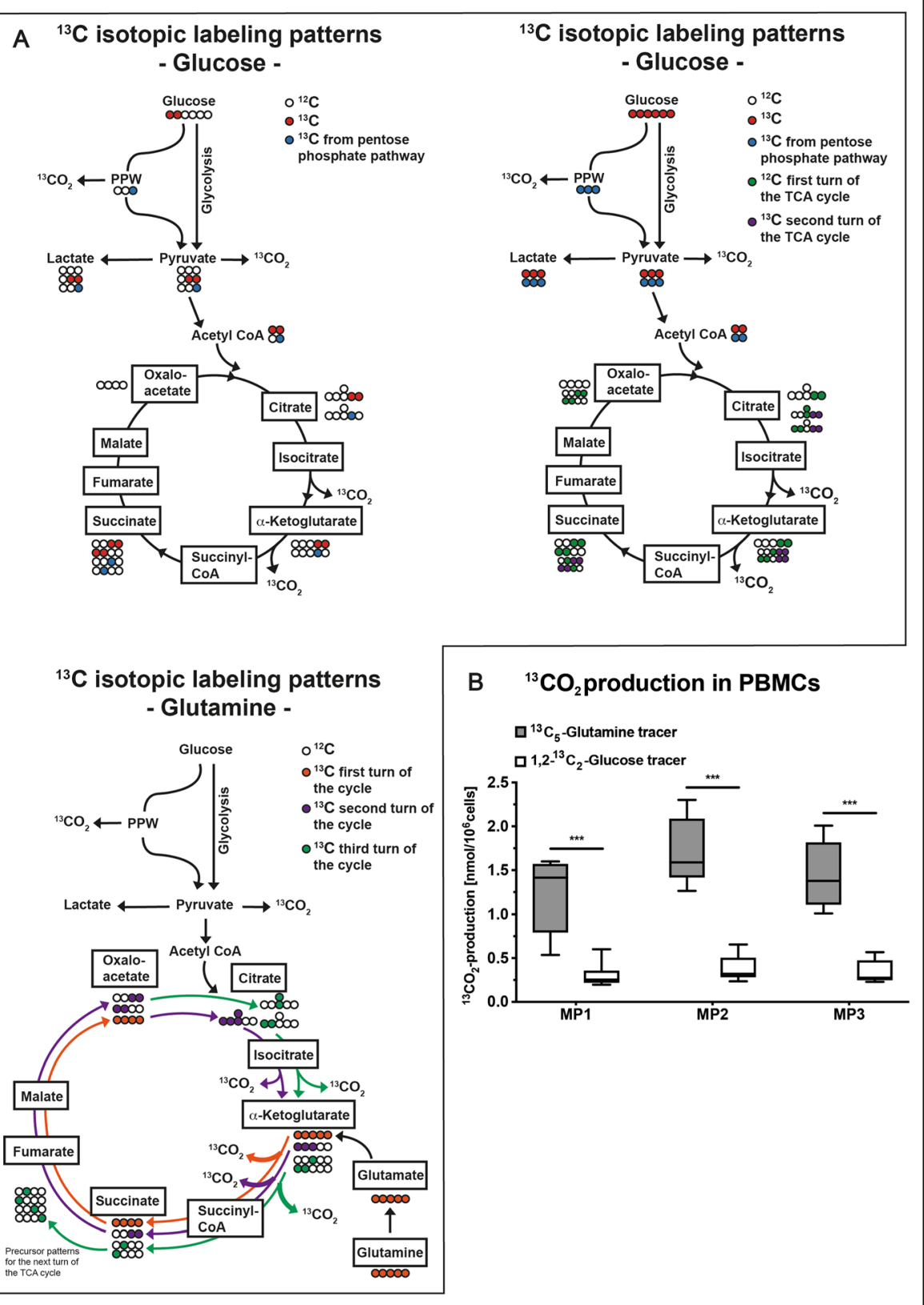

Fig. 3 Assessing cellular metabolism with stable isotope-labeled substrates. a ${ }^{13} \mathrm{C}$ labeling patterns of TCA cycle metabolites resulting from utilization of $1,2-{ }^{13} \mathrm{C}_{2}$-glucose (upper left), ${ }^{13} \mathrm{C}_{6}$-glucose (upper right), and ${ }^{13} \mathrm{C}_{5}$-glutamine (lower left). Depending on the substrate used, further conclusions can be drawn on the involvement of the PPP or the cycling within the TCA cycle. $\mathbf{b}{ }^{13} \mathrm{CO}_{2}$ production in PBMCs from a porcine model of acute subdural hematoma at MP before (MP1), $12 \mathrm{~h}$ (MP2), and $24 \mathrm{~h}$ (MP3) after hematoma induction. $5 \times 10^{6} \mathrm{PBMCs} / \mathrm{mL}$ were incubated in chemically identical RPMl media containing either $1,2-{ }^{13} \mathrm{C}_{2}$-glucose, ${ }^{13} \mathrm{C}_{6}$-glucose, or ${ }^{13} \mathrm{C}_{5}$-glutamine. Supernatant was transferred to airtight vials and acidified to release $\mathrm{CO}_{2}$ into the gas phase where ${ }^{13} \mathrm{CO}_{2}$ enrichment was determined by gas chromatography-mass spectrometry. Data are presented as median and interquartile range; ${ }^{* * *} p<0.0001$ according to a 2-way ANOVA and a Sidak's multiple comparisons test, $n=5-6$. Abbreviations: $\mathrm{CO}_{2}$, carbon dioxide; $\mathrm{MP}$, measurement timepoint; PBMCs, peripheral blood mononuclear cells; PPP, pentose phosphate pathway; TCA, tricarboxylic acid cycle 


\section{A Mitchondrial oxygen consumption}

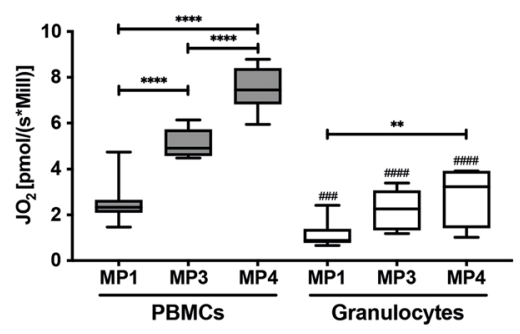

C Hydrogen peroxide production

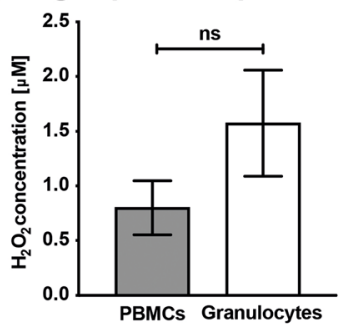

B Superoxide production
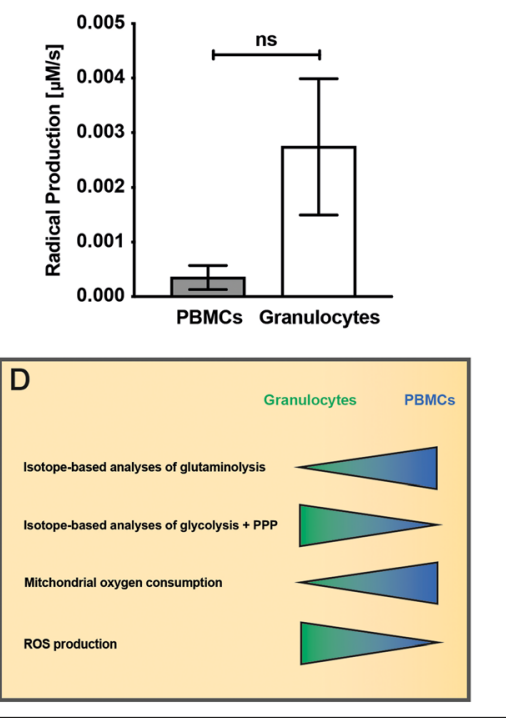

Fig. 4 Assessment of immune cell metabolism. Panel a shows the mitochondrial oxygen consumption in a porcine model of acute subdural hematoma combined with hemorrhagic shock assessed in the uncoupled state (electron transport system capacity) before (MP1), as well as 12 (MP2) and $24 \mathrm{~h}$ (MP3) after trauma induction in PBMCs (gray bars) and granulocytes (white bars). $10 \times 10^{6}$ cells $/ 1 \mathrm{~mL}$ RPMI medium were added to the measurement chamber of the Oxygraph O2K (Oroboros Instruments, Innsbruck, Austria) which measures oxygen concentration with a Clark electrode. After addition of the ATP synthase inhibitor oligomycine $(0.5 \mu \mathrm{L}$ of a $0.5 \mu \mathrm{M}$ stock solution), the uncoupling agent FCCP $(0.5 \mu \mathrm{L}$ of a $0.5 \mu \mathrm{M}$ stock solution) was added stepwise to reach the level of maximum oxygen consumption (electron transfer system capacity). $\mathbf{b} \mathrm{O}_{2}{ }^{-}$production quantified by electron spin resonance spectroscopy in PBMCs (gray bars) and granulocytes (white bars) isolated from pigs before trauma induction. Therefore, $2.5 \times 10^{6}$ cells (in $1 \mathrm{~mL}$ RPMI) were mixed with the superoxide-targeted spin probe $\mathrm{CMH}$. A serial measurement over $30 \mathrm{~min}$ enables calculation of superoxide radical production rate. An equally treated sample of RPMI with CMH served as blank value that was subtracted from the cell suspensions' production rate. c Before trauma induction, $\mathrm{H}_{2} \mathrm{O}_{2}$ production of $1 \times 10^{6}$ PBMCs (gray bars) and granulocytes (white bars) in $2 \mathrm{~mL}$ RPMl medium was electrochemically quantified with a Pt-black modified microelectrode. The data presented in b and $\mathbf{c}$ are adapted from [72]. $\mathbf{d}$ outlines the concept for our methodical approach to analyze the energy metabolism in granulocytes and PBMCs. Usually, granulocytes preferentially utilize glucose to produce ATP and have a low mitochondrial oxygen consumption but a high ROS production. PBMCs on the other hand prefer glutamine over glucose utilization, have a higher mitochondrial oxygen consumption and low ROS production. It is important to note that the metabolic pathways shift depending on the state of activation and that immune cells use different substrates (fatty acids, amino acids) in order to safeguard ATP homeostasis. Data are presented as a median with interquartile range and $\mathbf{b}, \mathbf{c}$ mean with $S D,{ }^{*} p<0.05$; ${ }^{* *} p$ $<0.01 ;{ }^{* * *} p<0.001 ;{ }^{* * * *} p<0.0001 ;{ }^{\# \# \#} p<0.001$ vs PBMC; ${ }^{\# \# \#} p<0.0001$ vs PBMC; according to a a 1 -way ANOVA and Tukey's multiple comparisons test, $n=6-14$, and $\mathbf{b}$ Wilcoxon signed-rank test, $n=7-9, \mathbf{c} n=3$ per group. Abbreviations: ATP, adenosine triphosphate; $\mathrm{CMH}$, 1-hydroxy-3-methoxycarbonyl-2,2,5,5tetramethylpyrrolidine (spin probe); FCCP, carbonyl cyanide-4-(trifluoromethoxy)phenylhydrazone (uncoupling agent); MP, measurement timepoint; PBMCs, peripheral blood mononuclear cells; Pt-black, platinum black; ROS, reactive oxygen species; SD, standard deviation

ROS production is crucial for both signaling and host defense [77-79]. Grondman et al. demonstrated this crucial role of ROS in human monocytes: ROS production was directly correlated to the percentage of microbial killing of Candida albicans [80]. The authors elegantly showed that enhanced ROS production was increased due to metabolic changes e.g. increased aerobic glycolysis, PPP, and oxidative burst. In monocytes of healthy volunteers intravenously challenged with LPS to simulate sepsis-induced immunoparalysis, killing capacity was reduced, which coincided with impaired ROS production and less marked metabolic changes [80, 81]. In other words, impaired 
monocyte "metabolic plasticity" following endotoxin-induced immunotolerance lead to decreased ROS release and, consecutively, impaired host defense capacity. As a result of the abovementioned difference in mitochondria content, granulocytes and monocytes not only show markedly different respiratory activity, but also ROS formation. Clearly, despite its fundamental role for host defense, increased granulocyte-derived ROS production may also have a "dark side": Kramer et al. reported in activated neutrophils that NADPH oxidase-derived $\mathrm{H}_{2} \mathrm{O}_{2}$ inhibited the metabolic shift of lymphocytes from OXPHOS to aerobic glycolysis, which was associated with decreased cytokine production as a mirror of depressed $\mathrm{T}_{\text {eff }}$ function [82].

Catecholamines have a profound impact on immune cell function [83, 84]. Their effect is dependent on the respective receptor stimulation: e.g., noradrenaline, the drug of choice for first-line hemodynamic support during septic shock, displays a pro-inflammatory profile when acting on $\alpha$-adrenoceptors by upregulating NF-kB, while $\beta$-adrenoceptor activation results in a more immunosuppressive pattern [84]. Vice versa, cells of the innate immune system, such as granulocytes and macrophages, are able to produce catecholamines, which may per se aggravate inflammatory responses [85].

Apart from their direct role on modulating inflammatory processes, catecholamines have also been associated with enhanced oxidative stress levels due to autooxidation [86, 87]. It is known from the literature that increased radical production can impair mitochondrial oxygen uptake. In line with this, Lünemann et al. showed that noradrenaline dose-dependently exerted anti-inflammatory effects by inhibiting mitochondrial function of PBMCs obtained from healthy blood donors [88].

\section{Immune cell bioenergetics and outcome}

Several authors showed in various pathological conditions that any impairment of immune cell oxygen consumption coincides with aggravated morbidity and mortality: Belikova et al. compared OXPHOS of PBMCs taken from healthy volunteers and patients with severe sepsis [89]. OXPHOS was higher in naïve PBMCs from patients with sepsis, but due to impaired responsiveness, this result was reversed upon stimulation with adenosine diphosphate (ADP). Incubation of healthy volunteer PBMCs with plasma from septic patients mimicked this finding [89]. In addition, Li et al. showed that mitochondrial oxygen consumption was reduced in PBMCs from patients with early-stage heart failure when compared to healthy volunteers [90], and Weiss et al. demonstrated mitochondrial dysfunction in circulating PBMCs during pediatric septic shock [91]. Cheng et al. used several approaches in order to assess the energy metabolism of immune cells in critical illness [92]. During the acute phase of the infection, the mTOR pathway orchestrated a shift from OXPHOS to glycolysis in PBMCs stimulated with either C. albicans or LPS (E. coli). In another approach, leukocytes obtained from either septic patients or healthy volunteers undergoing experimental endotoxemia showed a strong impairment of the cellular energy metabolism (glycolysis, mTOR signaling, OXPHOS, and FAO), that coincided with a decreased capacity to respond to secondary infection, also referred to as immunometabolic paralysis. This effect was partly reversed by the administration of interferon (INF)- $\gamma$, thereby underpinning its use in the treatment of sepsis [92].

In line with the abovementioned study, the assessment of energy metabolism of circulating immune cells may in fact provide a valuable approach for translational 
research, thereby modulating inflammatory responses or serving as a bioenergetic biomarker. However, before the energy metabolism can reliably be linked to pathophysiological states, more research has to be performed, particularly by precisely characterizing the energy metabolism of distinct subsets through more sophisticated approaches.

\section{Conclusion}

During an inflammatory response, immune cells are activated, undergo proliferation and differentiation, and exert key effector functions. This requires a continuous metabolic adaption.

Immune cell functions are tightly linked to metabolic programs and demonstrate metabolic alterations upon immune cell activation. Thus, inactivated immune cells are metabolically quiescent and shift to a higher metabolic level upon an immunological challenge. Immune cell energy metabolism can be further influenced by various factors: inflammatory responses can increase intracellular ROS levels. ROS are essential for various biological functions, such as reactive oxygen burst, however, also affect the energy metabolism. Besides, immune cell metabolism can be affected by a common therapeutic intervention, such as catecholamines, thereby reinforcing pro- or anti-inflammatory responses.

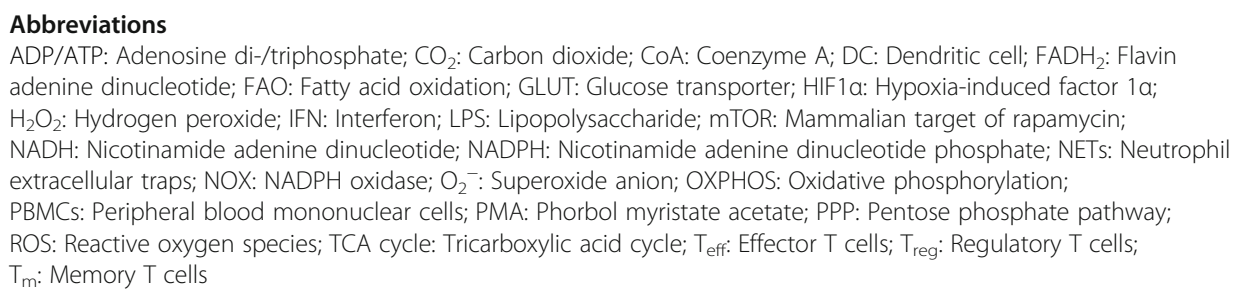

\section{Authors' contributions}

$\mathrm{CH}, \mathrm{PR}, \mathrm{MW}, \mathrm{BM}$, and $\mathrm{CK}$ conceived the review. $\mathrm{XZ}, \mathrm{FZ}, \mathrm{FH}, \mathrm{ML}$, and $\mathrm{AH}$ performed the literature review. $\mathrm{XZ}$ and $\mathrm{FZ}$ drafted the manuscript. UW, JV, CK, CH, BM, and PR revised the manuscript for important intellectual content, $\mathrm{CH}$ and PR critically reviewed and edited the manuscript. All authors were involved in the acquisition and interpretation of data and read and approved the final version.

\section{Funding}

PR and $\mathrm{CH}$ received funding from the DFG CRC 1149 (project number 251293561) and GRK 2203. FZ, AH, CK, and BM received funding from the DFG GRK 2203. XZ received funding from China Scholarship Council, Grant number: 201806160164. The funding bodies had no influence on the content of the manuscript. Publication costs are funded by DFG GRK 2203. Open access funding provided by Projekt DEAL. Open Access funding enabled and organized by Projekt DEAL.

Availability of data and materials

Data sharing is not applicable to this article as no datasets were generated or analyzed during the current study.

Ethics approval and consent to participate

Not applicable.

Consent for publication

Not applicable. 


\section{Author details}

${ }^{1}$ Institut für Anästhesiologische Pathophysiologie und Verfahrensentwicklung, Universitätsklinikum Ulm, Helmholzstraße 8/1, 89081 Ulm, Germany. ${ }^{2}$ Klinik für Anästhesiologie, Universitätsklinikum UIm, Ulm, Germany. ${ }^{3}$ Anesthesia and Intensive Care, San Martino Policlinico Hospital, IRCCS for Oncolocy and Neuroscience, Genoa, Italy. ${ }^{4}$ Institut für Analytische und Bioanalytische Chemie, Universität Ulm, Ulm, Germany.

Received: 7 June 2020 Accepted: 11 June 2020

Published online: 18 December 2020

\section{References}

1. Warburg O (1956) On the origin of cancer cells. Science (80- ) 123:309-314 https://doi.org/10.1126/science.123.3191.309

2. Epstein T, Gatenby RA, Brown JS (2017) The Warburg effect as an adaptation of cancer cells to rapid fluctuations in energy demand. PLoS One 12:1-14 https://doi.org/10.1371/journal.pone.0185085

3. Netea MG, Joosten LAB, Latz E et al (2016) Trained immunity: a program of innate immune memory in health and disease. Science (80- ) 352:aaf1098 https://doi.org/10.1126/science.aaf1098

4. Medzhitov R, Janeway C (2000) Innate immune recognition: mechanisms and pathways. Immunol Rev 173:89-97 https://doi.org/10.1034/j.1600-065X.2000.917309.x

5. Maianski NA, Maianski AN, Kuijpers TW, Roos D (2004) Apoptosis of Neutrophils. Acta Haematol 111:56-66 https://doi. org/10.1159/000074486

6. van Raam B, Verhoeven A, Kuijpers T (2006) Mitochondria in neutrophil apoptosis. Int J Hematol 84:199-204 https://doi. org/10.1532/IJH97.06131

7. Fossati G, Moulding DA, Spiller DG et al (2003) The mitochondrial network of human neutrophils: role in chemotaxis, phagocytosis, respiratory burst activation, and commitment to apoptosis. J Immunol 170:1964-1972 https://doi.org/10. 4049/jimmunol.170.4.1964

8. Karnovsky ML (1968) The metabolism of leukocytes. Semin Hematol 5:156-165

9. Sbarra AJ, Karnovskyi ML (1959) The Biochemical Basis of Phagocytosis I. Metabolic changes during the ingestion of particles by polymorphonuclear leukocytes. J Biol Chem 234:1355-1362

10. Kramer PA, Ravi S, Chacko B et al (2014) A review of the mitochondrial and glycolytic metabolism in human platelets and leukocytes: implications for their use as bioenergetic biomarkers. Redox Biol 2:206-210 https://doi.org/10.1016/j. redox.2013.12.026

11. Sumbayev W, Nicholas SA, Streatfield CL, Gibbs BF (2009) Involvement of hypoxia-inducible factor-1 HiF(1 a) in lgEmediated primary human basophil responses. Eur J Immunol 39:3511-3519 https://doi.org/10.1002/eji.200939370

12. Porter L, Toepfner N, Bashant KR et al (2018) Metabolic profiling of human eosinophils. Front Immunol 9. https://doi. org/10.3389/fimmu.2018.01404

13. Pearce EL, Pearce EJ (2013) Metabolic pathways in immune cell activation and quiescence. Immunity 38:633-643 https://doi.org/10.1016/j.immuni.2013.04.005

14. Bauer DE, Harris MH, Plas DR et al (2004) Cytokine stimulation of aerobic glycolysis in hematopoietic cells exceeds proliferative demand. 20:1303-1305

15. Watts ER, Walmsley SR (2019) Inflammation and hypoxia: HIF and PHD isoform selectivity. Trends Mol Med 25:33-46 https://doi.org/10.1016/j.molmed.2018.10.006

16. Semenzas GL, Roth PH, Fang H, Wang GL (1994) Transcriptional regulation of genes encoding glycolytic enzymes by hypoxia-inducible factor 1. J Biol Chem 269:23757-23763

17. Corcoran SE, O'Neill LAJ (2016) HIF1a and metabolic reprogramming in inflammation. J Clin Invest 126:3699-3707 https://doi.org/10.1172/JCl84431

18. Cramer T, Yamanishi Y, Clausen BE et al (2003) HIF-1a Is essential for myeloid cell-mediated inflammation. Cell 112:645657 https://doi.org/10.1016/S0092-8674(03)00154-5

19. Ben-Shoshan J, Maysel-Auslender S, Mor A et al (2008) Hypoxia controls CD4+ CD25+ regulatory T-cell homeostasis via hypoxia-inducible factor-1a. Eur J Immunol 38:2412-2418 https://doi.org/10.1002/eji.200838318

20. Kiers HD, Scheffer G-J, van der Hoeven JG et al (2016) Immunologic consequences of hypoxia during critical illness. Anesthesiology 125:237-249 https://doi.org/10.1097/ALN.0000000000001163

21. Maratou E, Dimitriadis G, Kollias A et al (2007) Glucose transporter expression on the plasma membrane of resting and activated white blood cells. Eur J Clin Invest 37:282-290 https://doi.org/10.1111/j.1365-2362.2007.01786.x

22. Rodríguez-Espinosa O, Rojas-Espinosa O, Moreno-Altamirano MMB et al (2015) Metabolic requirements for neutrophil extracellular traps formation. Immunology 145:213-224 https://doi.org/10.1111/imm.12437

23. Azevedo EP, Rochael NC, Guimarães-Costa AB et al (2015) A metabolic shift toward pentose phosphate pathway is necessary for amyloid fibril- and phorbol 12-myristate 13-Acetate-induced neutrophil extracellular trap (NET) formation. J Biol Chem 290:22174-22183 https://doi.org/10.1074/jbc.M115.640094

24. McInturff AM, Cody MJ, Elliott EA et al (2012) Mammalian target of rapamycin regulates neutrophil extracellular trap formation via induction of hypoxia-inducible factor 1 a. Blood 120:3118-3125 https://doi.org/10.1182/blood-2012-01 405993

25. Root RK, Metcalf JA (1977) H2O2 release from human granulocytes during phagocytosis: Relationship to superoxide anion formation and cellular catabolism of H2O2: Studies with normal and cytochalasin B-treated cells. J Clin Invest. https://doi.org/10.1172/JCl108886

26. Chen Y, Junger WG (2012) Measurement of oxidative burst in neutrophils. In: Ashman RB (ed) Methods in molecular biology (Clifton, N.J.). Humana Press, Totowa, pp 115-124

27. Dale DC, Boxer L, Liles WC (2008) The phagocytes: neutrophils and monocytes. Blood 112:935-945 https://doi.org/10. 1182/blood-2007-12-077917

28. Guthrie LA, McPhail LC, Henson PM, Johnston RB (1984) Priming of neutrophils for enhanced release of oxygen metabolites by bacterial lipopolysaccharide. Evidence for increased activity of the superoxide-producing enzyme. J Exp Med 160:1656-1671 https://doi.org/10.1084/jem.160.6.1656 
29. Newsholme P, Curi R, Pithon Curi TC et al (1999) Glutamine metabolism by lymphocytes, macrophages, and neutrophils: its importance in health and disease. J Nutr Biochem 10:316-324 https://doi.org/10.1016/S0955-2863(99)00022-4

30. Curi R, Newsholme P, Pithon-Curi TC et al (1999) Metabolic fate of glutamine in lymphocytes, macrophages and neutrophils. Brazilian J Med Biol Res 32:15-21 https://doi.org/10.1590/S0100-879X1999000100002

31. Riffelmacher T, Clarke A, Richter FC et al (2017) Autophagy-dependent generation of free fatty acids is critical for normal neutrophil differentiation. Immunity 47:466-480.e5 https://doi.org/10.1016/j.immuni.2017.08.005

32. Rodríguez-Prados J-C, Través PG, Cuenca J et al (2010) Substrate fate in activated macrophages: a comparison between innate, classic, and alternative activation. J Immunol 185:605-614 https://doi.org/10.4049/jimmunol.0901698

33. Serbina NV, Jia T, Hohl TM, Pamer EG (2008) Monocyte-mediated defense against microbial pathogens. Annu Rev Immunol 26:421-452 https://doi.org/10.1146/annurev.immunol.26.021607.090326

34. Dietl K, Renner K, Dettmer K et al (2010) Lactic acid and acidification inhibit TNF secretion and glycolysis of human monocytes. J Immunol 184:1200-1209 https://doi.org/10.4049/jimmunol.0902584

35. Martinez FO, Gordon S (2014) The M1 and M2 paradigm of macrophage activation: time for reassessment. F1000Prime Rep 6:13 https://doi.org/10.12703/P6-13

36. Odegaard Jl, Chawla A (2011) Alternative macrophage activation and metabolism. Annu Rev Pathol Mech Dis 6:275-297 https://doi.org/10.1146/annurev-pathol-011110-130138

37. Krawczyk CM, Holowka T, Sun J et al (2010) Toll-like receptor-induced changes in glycolytic metabolism regulate dendritic cell activation. Blood 115:4742-4749 https://doi.org/10.1182/blood-2009-10-249540

38. Newsholme P, Curi R, Gordon S, Newsholme EA (1986) Metabolism of glucose, glutamine, long-chain fatty acids and ketone bodies by murine macrophages. Biochem J 239:121-125 https://doi.org/10.1042/bj2390121

39. Wang T, Liu H, Lian G et al (2017) HIF1 a -induced glycolysis metabolism is essential to the activation of inflammatory macrophages. Mediators Inflamm. https://doi.org/10.1155/2017/9029327

40. Newsholme P, Newsholme EA (1989) Rates of utilization of glucose, glutamine and oleate and formation of endproducts by mouse perioneal macrophages in culture. Biochem J. https://doi.org/10.1042/bj2610211

41. Galván-Peña S, O’Neill LAJ (2014) Metabolic reprogramming in macrophage polarization. Front Immunol 5:1-6 https:// doi.org/10.3389/fimmu.2014.00420

42. Vats D, Mukundan L, Odegaard Jl et al (2006) Oxidative metabolism and PGC-1 $\beta$ attenuate macrophage-mediated inflammation. Cell Metab 4:13-24 https://doi.org/10.1016/j.cmet.2006.05.011

43. Zhu X, Meyers A, Long D et al (2019) Frontline science: monocytes sequentially rewire metabolism and bioenergetics during an acute inflammatory response. J Leukoc Biol 105:215-228 https://doi.org/10.1002/JLB.3HI0918-373R

44. Patil NK, Bohannon JK, Sherwood ER (2019) Metabolism drives monocytes during inflammation: What we do and do not know. J Leukoc Biol 105:211-213 https://doi.org/10.1002/JLB.3CE1118-448R

45. Arts RJW, Novakovic B, ter Horst R et al (2016) Glutaminolysis and fumarate accumulation integrate immunometabolic and epigenetic programs in trained immunity. Cell Metab 24:807-819 https://doi.org/10.1016/j.cmet.2016.10.008

46. Cheng S-C, Quintin J, Cramer RA et al (2014) mTOR- and HIF-1 -mediated aerobic glycolysis as metabolic basis for trained immunity. Science (80- ) 345:1250684 https://doi.org/10.1126/science.1250684

47. Jones N, Vincent EE, Cronin JG et al (2019) Akt and STAT5 mediate naïve human CD4+ T-cell early metabolic response to TCR stimulation. Nat Commun. https://doi.org/10.1038/s41467-019-10023-4

48. Almeida L, Lochner M, Berod L, Sparwasser T (2016) Metabolic pathways in T cell activation and lineage differentiation. Semin Immunol 28:514-524 https://doi.org/10.1016/j.smim.2016.10.009

49. Buck MD, O'Sullivan D, Pearce EL (2015) T cell metabolism drives immunity. J Exp Med 212:1345-1360 https://doi.org/10. 1084/jem.20151159

50. Loftus RM, Finlay DK (2016) Immunometabolism: cellular metabolism turns immune regulator. J Biol Chem 291:1-10 https://doi.org/10.1074/jbc.R115.693903

51. Shi LZ, Wang R, Huang G et al (2011) HIFla-dependent glycolytic pathway orchestrates a metabolic checkpoint for the differentiation of TH17 and Treg cells. J Exp Med 208:1367-1376 https://doi.org/10.1084/jem.20110278

52. Pearce EL, Poffenberger MC, Chang C-H, Jones RG (2013) Fueling immunity: insights into metabolism and lymphocyte function. Science (80- ) 342:1242454 https://doi.org/10.1126/science.1242454

53. Vander Heiden MG, Cantley LC, Thompson CB (2009) Understanding the Warburg effect: the metabolic requirements of cell proliferation. Science (80- ) 324:1029-1033 https://doi.org/10.1126/science.1160809

54. Maclver NJ, Michalek RD, Rathmell JC (2013) Metabolic regulation of T lymphocytes. Annu Rev Immunol 31:259-283 https://doi.org/10.1146/annurev-immunol-032712-095956

55. Pearce EL (2010) Metabolism in T cell activation and differentiation. Curr Opin Immunol 22:314-320 https://doi.org/10. 1016/j.coi.2010.01.018

56. Chang C-H, Curtis JD, Maggi LB et al (2013) Posttranscriptional control of T cell effector function by aerobic glycolysis. Cell 153:1239-1251 https://doi.org/10.1016/j.cell.2013.05.016

57. Gubser PM, Bantug GR, Razik L et al (2013) Rapid effector function of memory CD8+ T cells requires an immediate-early glycolytic switch. Nat Immunol 14:1064-1072 https://doi.org/10.1038/ni.2687

58. Michalek RD, Gerriets VA, Jacobs SR et al (2011) Cutting edge: distinct glycolytic and lipid oxidative metabolic programs are essential for effector and regulatory CD4 + T cell subsets. J Immunol 186:3299-3303 https://doi.org/10.4049/ jimmunol.1003613

59. Angelin A, Gil-de-Gómez L, Dahiya S et al (2017) Foxp3 reprograms T cell metabolism to function in low-glucose, highlactate environments. Cell Metab 25:1282-1293.e7 https://doi.org/10.1016/j.cmet.2016.12.018

60. Gerriets VA, Kishton RJ, Johnson MO et al (2016) Foxp3 and Toll-like receptor signaling balance T reg cell anabolic metabolism for suppression. Nat Immunol 17:1459-1466 https://doi.org/10.1038/ni.3577

61. van der Windt GJW, Everts B, Chang C-H et al (2012) Mitochondrial respiratory capacity is a critical regulator of CD8+ T cell memory development. Immunity 36:68-78 https://doi.org/10.1016/j.immuni.2011.12.007

62. Boothby M, Rickert RC (2017) Metabolic regulation of the immune humoral response. Immunity 46:743-755 https://doi. org/10.1016/j.immuni.2017.04.009

63. Khalsa JK, Chawla AS, Prabhu SB et al (2019) Functionally significant metabolic differences between B and T lymphocyte lineages. Immunology 158:104-120 https://doi.org/10.1111/imm.13098 
64. Doughty CA, Bleiman BF, Wagner DJ et al (2006) Antigen receptor-mediated changes in glucose metabolism in B lymphocytes: role of phosphatidylinositol 3-kinase signaling in the glycolytic control of growth. Blood 107:4458-4465 https://doi.org/10.1182/blood-2005-12-4788

65. Dufort FJ, Bleiman BF, Gumina MR et al (2007) Cutting edge: IL-4-mediated protection of primary B lymphocytes from apoptosis via Stat6-dependent regulation of glycolytic metabolism. J Immunol 179:4953-4957 https://doi.org/10.4049/ jimmunol.179.8.4953

66. Limon JJ, Fruman DA (2012) Akt and mTOR in B cell activation and differentiation. Front Immunol 3:1-12 https://doi. org/10.3389/fimmu.2012.00228

67. Waters LR, Ahsan FM, Wolf DM et al (2018) Initial B cell activation induces metabolic reprogramming and mitochondrial remodeling. iScience 5:99-109 https://doi.org/10.1016/j.isci.2018.07.005

68. Ahn WS, Antoniewicz MR (2013) Parallel labeling experiments with [1,2-13C]glucose and [U-13C]glutamine provide new insights into CHO cell metabolism. Metab Eng 15:34-47 https://doi.org/10.1016/j.ymben.2012.10.001

69. Vogt JA, Yarmush DM, Yu YM et al (1997) TCA cycle flux estimates from NMR- and GC-MS-determined [13C]glutamate isotopomers in liver. Am J Physiol 272:C2049-C2062 https://doi.org/10.1152/ajpcell.1997.272.6.C2049

70. Antoniewicz MR, Kelleher JK, Stephanopoulos G (2007) Elementary metabolite units (EMU): a novel framework for modeling isotopic distributions. Metab Eng 9:68-86 https://doi.org/10.1016/j.ymben.2006.09.001

71. Buescher JM, Antoniewicz MR, Boros LG et al (2015) A roadmap for interpreting 13 C metabolite labeling patterns from cells. Curr Opin Biotechnol 34:189-201 https://doi.org/10.1016/j.copbio.2015.02.003

72. Hellmann A, Daboss S, Zink F et al (2020) Electrocatalytically modified microelectrodes for the detection of hydrogen peroxide at blood cells from swine with induced trauma. Electrochim Acta 136458 https://doi.org/10.1016/j.electacta. 2020.136458

73. Datzmann T, Kapapa T, Scheuerle A et al (2019) In-depth characterization of a long-term, resuscitated model of acute subdural hematoma-induced brain injury. J Neurosurg:1-12 https://doi.org/10.3171/2019.9.JNS191789

74. Boveris A, Chance B (1973) The mitochondrial generation of hydrogen peroxide. General properties and effect of hyperbaric oxygen. Biochem J 134:707-716 https://doi.org/10.1042/bj1340707

75. Wong H-S, Dighe PA, Mezera $V$ et al (2017) Production of superoxide and hydrogen peroxide from specific mitochondrial sites under different bioenergetic conditions. J Biol Chem 292:16804-16809 https://doi.org/10.1074/jbc. R117.789271

76. Hill BG, Benavides GA, Lancaster JR et al (2012) Integration of cellular bioenergetics with mitochondrial quality control and autophagy. Biol Chem 393:1485-1512 https://doi.org/10.1515/hsz-2012-0198

77. Magder S (2006) Reactive oxygen species: toxic molecules or spark of life? Crit Care 10:1-8 https://doi.org/10. $1186 /$ cc3992

78. Winterbourn CC, Kettle AJ, Hampton MB (2016) Reactive oxygen species and neutrophil function. Annu Rev Biochem 85:765-792 https://doi.org/10.1146/annurev-biochem-060815-014442

79. Nguyen GT, Green ER, Mecsas J (2017) Neutrophils to the ROScue: Mechanisms of NADPH oxidase activation and bacterial resistance. Front Cell Infect Microbiol 7. https://doi.org/10.3389/fcimb.2017.00373

80. Grondman I, Arts RJW, Koch RM et al (2019) Endotoxin-induced immunotolerance is associated with loss of monocyte metabolic plasticity and reduction of oxidative burst. J Leukoc Biol 106:11-25 https://doi.org/10.1002/JLB.5HI0119-018R

81. Williams DL, Li C, Sherwood ER (2019) Loss of monocyte metabolic plasticity in endotoxin tolerance: a model for understanding sepsis-induced immune paralysis? J Leukoc Biol 106:7-9 https://doi.org/10.1002/JLB.4CE0319-100R

82. Kramer PA, Prichard L, Chacko B et al (2015) Inhibition of the lymphocyte metabolic switch by the oxidative burst of human neutrophils. Clin Sci 129:489-504 https://doi.org/10.1042/CS20140852

83. Hartmann C, Radermacher P, Wepler M, Nußbaum B (2017) Non-hemodynamic effects of catecholamines. SHOCK 48: 390-400 https://doi.org/10.1097/SHK.0000000000000879

84. Stolk RF, Van Der Poll T, Angus DC et al (2016) Potentially inadvertent immunomodulation: norepinephrine use in sepsis. Am J Respir Crit Care Med 194:550-558 https://doi.org/10.1164/rccm.201604-0862CP

85. Flierl MA, Rittirsch D, Nadeau BA et al (2007) Phagocyte-derived catecholamines enhance acute inflammatory injury. Nature 449:721-725 https://doi.org/10.1038/nature06185

86. Rump AFE, Klaus W (1994) Evidence for norepinephrine cardiotoxicity mediated by superoxide anion radicals in isolated rabbit hearts. Naunyn Schmiedebergs Arch Pharmacol 349:295-300 https://doi.org/10.1007/BF00169296

87. Neri M, Cerretani D, Fiaschi Al et al (2007) Correlation between cardiac oxidative stress and myocardial pathology due to acute and chronic norepinephrine administration in rats. J Cell Mol Med 11:156-170 https://doi.org/10.1111/j.15824934.2007.00009.x

88. Lünemann J, Buttgereit F, Tripmacher R et al (2002) Norepinephrine inhibits energy metabolism of human peripheral blood mononuclear cells via adrenergic receptors. Biosci Rep 22:627-635 https://doi.org/10.1023/A:1020155126321

89. Belikova I, Lukaszewicz AC, Faivre V et al (2007) Oxygen consumption of human peripheral blood mononuclear cells in severe human sepsis. Crit Care Med 35:2702-2708 https://doi.org/10.1097/01.CCM.0000295593.25106.C4

90. Li P, Wang B, Sun F et al (2015) Mitochondrial respiratory dysfunctions of blood mononuclear cells link with cardiac disturbance in patients with early-stage heart failure. Sci Rep 5:10229 https://doi.org/10.1038/srep10229

91. Weiss SL, Selak MA, Tuluc F et al (2015) Mitochondrial dysfunction in peripheral blood mononuclear cells in pediatric septic shock. Pediatr Crit Care Med 16:e4-e12 https://doi.org/10.1097/PCC.0000000000000277

92. Cheng S-C, Scicluna BP, Arts RJW et al (2016) Broad defects in the energy metabolism of leukocytes underlie immunoparalysis in sepsis. Nat Immunol 17:406-413 https://doi.org/10.1038/ni.3398

\section{Publisher's Note}

Springer Nature remains neutral with regard to jurisdictional claims in published maps and institutional affiliations. 